\title{
Choice on the simplex domain
}

Citation for published version (APA):

Bossert, W., \& Peters, H. (2017). Choice on the simplex domain. Maastricht University, Graduate School of Business and Economics. GSBE Research Memoranda No. 030

https://doi.org/10.26481/umagsb.2017030

Document status and date:

Published: 05/12/2017

DOI:

10.26481/umagsb.2017030

Document Version:

Publisher's PDF, also known as Version of record

\section{Please check the document version of this publication:}

- A submitted manuscript is the version of the article upon submission and before peer-review. There can be important differences between the submitted version and the official published version of record.

People interested in the research are advised to contact the author for the final version of the publication, or visit the DOI to the publisher's website.

- The final author version and the galley proof are versions of the publication after peer review.

- The final published version features the final layout of the paper including the volume, issue and page numbers.

Link to publication

\footnotetext{
General rights rights.

- You may freely distribute the URL identifying the publication in the public portal. please follow below link for the End User Agreement:

www.umlib.nl/taverne-license

Take down policy

If you believe that this document breaches copyright please contact us at:

repository@maastrichtuniversity.nl

providing details and we will investigate your claim.
}

Copyright and moral rights for the publications made accessible in the public portal are retained by the authors and/or other copyright owners and it is a condition of accessing publications that users recognise and abide by the legal requirements associated with these

- Users may download and print one copy of any publication from the public portal for the purpose of private study or research.

- You may not further distribute the material or use it for any profit-making activity or commercial gain

If the publication is distributed under the terms of Article $25 \mathrm{fa}$ of the Dutch Copyright Act, indicated by the "Taverne" license above, 


\section{Maastricht University}

Walter Bossert, Hans Peters

Choice on the simplex domain

$\mathrm{RM} / 17 / 030$

\section{GSBE}

Maastricht University School of Business and Economics

Graduate School of Business and Economics

P.O Box 616

NL- 6200 MD Maastricht

The Netherlands 


\title{
Choice on the simplex domain*
}

\author{
WALTER BOSSERT \\ Centre Interuniversitaire de Recherche en \\ Economie Quantitative (CIREQ) \\ P.O. Box 6128, Station Downtown \\ Montreal QC H3C 3J7 \\ Canada \\ e-mail: walter.bossert@videotron.ca \\ and \\ HANS PETERS \\ Department of Quantitative Economics, Maastricht University \\ P.O. Box 616 \\ 6200 MD Maastricht, The Netherlands \\ h.peters@maastrichtuniversity.nl
}

This version: November 21, 2017

\begin{abstract}
One unit of a good has to be divided among a group $N$ of individuals who each are entitled to a minimal share and these shares sum up to less than one. The associated set of choice problems consists of the unit simplex and all its full-dimensional subsimplices with the same orientation. We characterize all choice rules that are independent of irrelevant alternatives, continuous, and monotonic. The resulting rules are what we refer to as $N$ path choice functions. If there are only three individuals, the monotonicity property can be weakened. We also consider the issue of rationalizability and show that, for the threeagent case, excluding cycles of length three in the revealed preference relation implies the strong axiom of revealed preference, that is, the exclusion of cycles of any length. Journal of Economic Literature Classification Nos.: D11, D71.
\end{abstract}

Keywords. Choice functions, simplex domain, rationalizability.

* Financial support from the Research School METEOR of Maastricht University, the Dutch Science Foundation NWO (grant no. 040.11.320), the Fonds de Recherche sur la Société et la Culture of Québec, and the Social Sciences and Humanities Research Council of Canada is gratefully acknowledged. 


\section{Introduction}

In this paper we consider the following division problem: one unit of a good (for example, money) is to be divided among $n$ individuals who are entitled to a minimal share of this good, where these shares sum up to less than one; in other words, there is a surplus. Thus, the set of division problems consists of the unit simplex and all its full-dimensional subsimplices with the same orientation. Our approach to this division problem is choice-theoretic: we interpret a chosen division as the best one among all feasible divisions, and therefore assume that it should again be chosen if it is still available when the minimal entitlements increase. Expressed more formally, we consider choice rules that are independent of irrelevant alternatives in the sense of Nash (1950). The other basic property we impose on choice rules is continuity with respect to the minimal shares. Combined, these two conditions have an additional powerful consequence: if an individual receives more than the minimal share in a certain division problem and this minimal share decreases, then the chosen division does not change (Lemma 1). We use the terms division problem and choice problem interchangeably in this paper.

For the general $n$-person choice problem we add a third condition, called monotonicity. Suppose that, in a given division problem, an individual receives its minimal share at a chosen division. If this minimal share increases, the additional amount accruing to this individual is taken away only from other individuals who initially received more than their minimal shares. This is a plausible fairness condition and facilitates the description of possible choice rules. Our main result (Theorems 1 and 2 in Section 3) is the characterization of all choice rules satisfying independence of irrelevant alternatives, continuity, and monotonicity. We refer to the resulting choice rules as $N$-path choice functions, where $N$ is the set of individuals. An $N$-path is a collection of monotonic and continuous curves for $N$ and for all subsets of $N$, in the simplex that represents the choice problem when all minimal shares are zero. These curves additionally satisfy a consistency condition. The associated rule assigns to the subsimplex corresponding to a choice problem the point where one of these curves enters the subsimplex; consistency ensures that if two different curves enter the subsimplex, this entry point is independent of the choice of the requisite curve. For the case $n=3$ we obtain a larger class of rules by weakening the monotonicity condition (Theorem 3).

In the final part of the paper (Section 5) we investigate the issue of rationalizability. In our setting, independence of irrelevant alternatives is equivalent to the weak axiom of revealed preference, which requires that in the preference relation induced by a choice rule there are no cycles of length two. The strong axiom of revealed preference demands that there are no cycles of any length. We first show that, in the three-agent case, excluding cycles of length $K$ or less does not necessarily exclude cycles of length $K+1$ (Theorem 4) - thus, in particular, independence of irrelevant alternatives does not guarantee rationalizability, that is, the strong axiom of revealed preference. Second, we show that (again, for the case of three individuals) adding continuity and non-existence of cycles of length three implies the strong axiom of revealed preference (Theorem 5). An extension of the latter result for $n>3$ remains an open question.

The choice problem examined in this paper can be seen as the counterpart of the 
familiar bankruptcy problem, where the shares are claims which sum up to more than one and thus cannot all be satisfied. See Thomson (2015) for an up-to-date overview of the literature on bankruptcy problems. Stovall (2014) considers the bankruptcy problem from a choice-theoretic perspective similar to ours.

Our paper contributes to the literature on revealed preference and rationalizability, starting with the seminal work of Samuelson (1938) and later Arrow (1959), Uzawa (1960), and Richter $(1966,1971)$. We refer to Bossert and Suzumura (2010) for a recent overview of this literature. The present paper is perhaps most closely related to Bossert and Peters (2009), where choice sets are compact and convex subsets of $\mathbb{R}^{n}$. Here, the domain of choice sets - the standard simplex in $\mathbb{R}^{n}$ and all its subsimplices with the same orientation-is on the one hand much smaller but on the other hand much more specified. One of the common denominators in the literature (see, for instance, Rose, 1958, Peters and Wakker, 1991; 1994, Bossert, 1994, Blackorby, Bossert, and Donaldson, 1995, and Bossert and Peters, 2009 ) is that while in two dimensions (which corresponds to the case $n=3$ in the present paper) cycles of any length are excluded if cycles of length two or three are excluded, this observation does not carry over to more than two dimensions. Thus, as mentioned above, the case of more than three agents remains an unsolved problem.

\section{Basic definitions}

We consider $n$-dimensional choice problems, where $n \in \mathbb{N} \backslash\{1\}$. The set of coordinates is $N=\{1, \ldots, n\}$. Our notation for vector inequalities is $x \geq y$ if $x_{i} \geq y_{i}$ for all $i \in N$ and $x>y$ if $x_{i}>y_{i}$ for all $i \in N$. The Euclidean distance is denoted by $\|\cdot\|$ and $e^{i}$ is the $i^{t h}$ unit vector. We adopt the notational convention $\sum_{j \in \emptyset} p_{j}=0$.

The elements of the set

$$
A=\left\{\alpha \in \mathbb{R}_{+}^{n} \mid \sum_{i=1}^{n} \alpha_{i}<1\right\}
$$

are used to parametrize the choice problems considered in this paper. For any $\alpha \in A$, the feasible set corresponding to $\alpha$ is given by

$$
\Delta(\alpha)=\left\{x \in \mathbb{R}^{n} \mid x \geq \alpha \text { and } \sum_{i=1}^{n} x_{i}=1\right\} .
$$

Observe that each $\Delta(\alpha)$ is a full-dimensional subsimplex of the unit simplex in $\mathbb{R}^{n}$ with the same orientation. It represents the division problem where each individual $i$ is entitled to a minimal share $\alpha_{i}$. For $\alpha \in A$ and $i \in N$, the vector that is obtained by replacing $\alpha_{i}$ with $\alpha_{i}^{\prime}$ is denoted by $\left(\alpha_{-i}, \alpha_{i}^{\prime}\right)$.

A choice function is a mapping $C: A \rightarrow \Delta(0, \ldots, 0)$ such that $C(\alpha) \geq \alpha$ for all $\alpha \in A$. We interpret $C(\alpha)$ as the choice from the simplex $\Delta(\alpha)$. Note that we restrict attention to single-valued choices.

The following properties of choice functions are used in this paper. 
Independence of irrelevant alternatives. For all $\alpha, \beta \in A$,

$$
[C(\alpha) \geq \beta \geq \alpha] \Rightarrow C(\beta)=C(\alpha) .
$$

Continuity. For all sequences $\left\langle\alpha^{q}\right\rangle_{q \in \mathbb{N}}$ such that $\alpha^{q} \in A$ for all $q \in \mathbb{N}$ and all $\alpha \in A$,

$$
\lim _{q \rightarrow \infty} \alpha^{q}=\alpha \Rightarrow \lim _{q \rightarrow \infty} C\left(\alpha^{q}\right)=C(\alpha) .
$$

Monotonicity. For all $\alpha \in A$, all $i \in N$ and $\alpha_{i}^{\prime} \in\left(\alpha_{i}, 1\right)$ such that $\left(\alpha_{-i}, \alpha_{i}^{\prime}\right) \in A$, and all $j \in N$ such that $C(\alpha)_{j}>\alpha_{j}$,

$$
C(\alpha)_{i}=\alpha_{i} \Rightarrow C\left(\alpha_{-i}, \alpha_{i}^{\prime}\right)_{j} \leq C(\alpha)_{j} .
$$

Independence of irrelevant alternatives and continuity are well-established properties in choice theory. Monotonicity says that if the $i^{\text {th }}$ constraint at a point $\alpha \in A$ is active at the choice from $\Delta(\alpha)$ and this constraint is increased, then the choice in the new situation cannot increase at any other constraint where there was slack before. In other words, if individual $i$ obtains its minimal share and in the new choice (division) problem this minimal share increases, so that individual $i$ will receive more, then no other individual who received more than its minimal share originally, will be better off in the new problem. This is a plausible property for a choice rule, and it will facilitate the description of the class of $N$-path choice rules in the next section.

The following lemma says that under independence of irrelevant alternatives and continuity, if an individual receives more than its minimal share, then the chosen outcome will not change if this minimal share decreases. The lemma will be extremely useful throughout the paper.

Lemma 1. Let $n \in \mathbb{N} \backslash\{1\}$, let $C$ be a choice function satisfying independence of irrelevant alternatives and continuity, and let $\alpha \in A, i \in N$ and $t_{i} \in\left[0, \alpha_{i}\right)$. If $\alpha_{i}<C(\alpha)_{i}$, then $C(\alpha)=C\left(\alpha_{-i}, t_{i}\right)$.

Proof. By way of contradiction, suppose that $C(\alpha) \neq C\left(\alpha_{-i}, t_{i}\right)$. By continuity, there exists $t_{i}^{\prime} \in\left[t_{i}, \alpha_{i}\right)$ such that

$$
C\left(\alpha_{-i}, t_{i}^{\prime}\right) \geq \alpha \text { and } C\left(\alpha_{-i}, t_{i}^{\prime}\right) \neq C(\alpha) .
$$

Since $C\left(\alpha_{i}, t_{i}^{\prime}\right) \geq \alpha \geq\left(\alpha_{-i}, t_{i}^{\prime}\right)$, independence of irrelevant alternatives implies that

$$
C\left(\alpha_{-i}, t_{i}^{\prime}\right)=C(\alpha)
$$

which is a contradiction.

Lemma 1 is illustrated for $n=3$ in Figure 1 . Let $\alpha=\left(0, \frac{1}{2}, \frac{1}{8}\right)$ and $y=\left(\frac{1}{4}, \frac{1}{2}, \frac{1}{4}\right)=C\left(0, \frac{1}{2}, \frac{1}{8}\right)$. We have $\alpha_{3}=\frac{1}{8}<\frac{1}{4}=y_{3}$. Let $t_{3}=0$ and suppose, by way of contradiction, that $C\left(0, \frac{1}{2}, 0\right)=z=\left(\frac{7}{16}, \frac{1}{2}, \frac{1}{16}\right) \neq y$. Continuity implies that there exists $t_{3}^{\prime} \in\left[0, \frac{1}{8}\right)$ such that

$$
C\left(0, \frac{1}{2}, t_{3}^{\prime}\right) \geq \alpha \text { and } C\left(0, \frac{1}{2}, t_{3}^{\prime}\right)=z^{\prime} \neq y \text {. }
$$


But we have $\left(0, \frac{1}{2}, \frac{1}{8}\right) \leq\left(0, \frac{1}{2}, t_{3}^{\prime}\right)$ and independence of irrelevant alternatives implies that $C\left(0, \frac{1}{2}, \frac{1}{8}\right)=C\left(0, \frac{1}{2}, t_{3}^{\prime}\right)$. This contradicts $C\left(0, \frac{1}{2}, \frac{1}{8}\right)=y$.

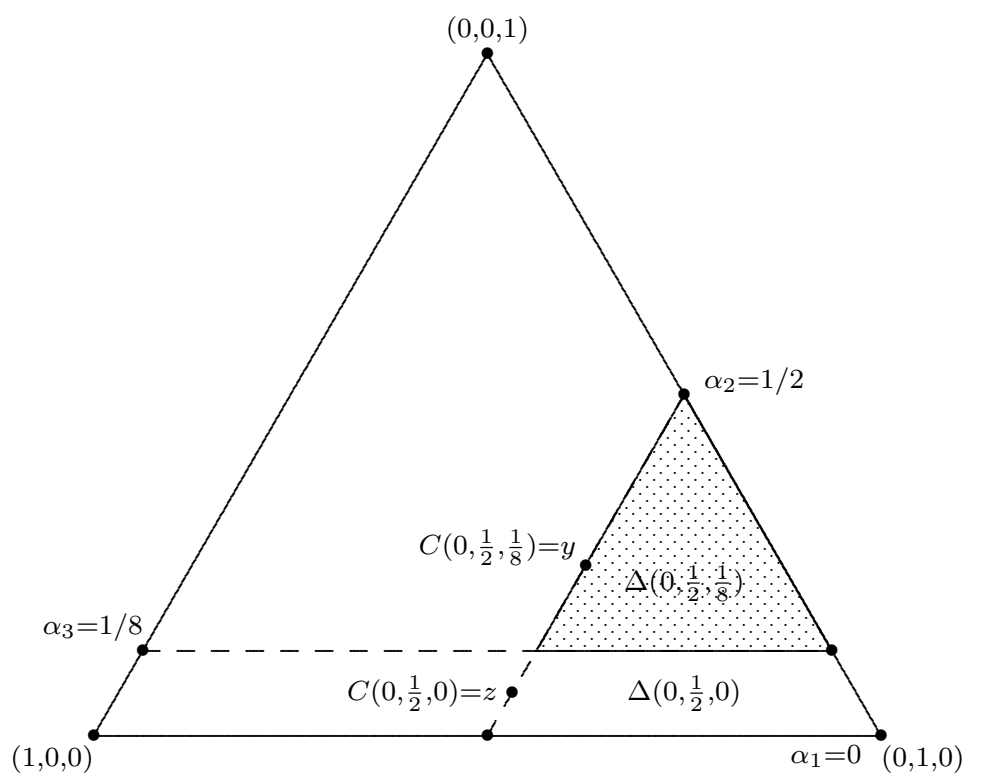

Figure 1: Proof of Lemma 1.

\section{$3 \quad N$-path choice functions}

In this section we define $N$-path choice functions and show that these are exactly all choice functions satisfying independence of irrelevant alternatives, continuity, and monotonicity. We first define elementary $S$-paths (Definition 1), then $N$-paths (Definition 2), and finally $N$-path choice functions (Definition 3).

\section{Definition 1.}

(a) Let $S \subseteq N$ with $|S| \geq 2$. An elementary $S$-path is a $(1+|S|)$-tuple $\left(p,\left(f^{i}\right)_{i \in S}\right)$ where

(i) $p \in \Delta(0, \ldots, 0)$ is such that $\sum_{j \in N \backslash S} p_{j}<1$;

(ii) for each $i \in S, f^{i}:\left[p_{i}, 1-\sum_{j \in N \backslash S} p_{j}\right] \rightarrow\left\{x \in \Delta(0, \ldots, 0) \mid x_{j}=p_{j}\right.$ for all $\left.j \in N \backslash S\right\}$ is a continuous function such that $f^{i}\left(t_{i}\right)=t_{i}$ for each $t_{i} \in\left[p_{i}, 1-\sum_{j \in N \backslash S} p_{j}\right]$.

(b) An elementary $S$-path $\left(p,\left(f^{i}\right)_{i \in S}\right)$ is monotonic if, for all $i \in S$ and all $t_{i}, t_{i}^{\prime} \in$ $\left[p_{i}, 1-\sum_{j \in N \backslash S} p_{j}\right], t_{i}^{\prime}>t_{i}$ implies $f^{i}\left(t_{i}^{\prime}\right)_{j} \leq f^{i}\left(t_{i}\right)_{j}$ for all $j \in S \backslash\{i\}$.

Thus, an elementary $S$-path is obtained by fixing the coordinates of individuals outside $S$, picking a point in the subsimplex for $S$, and starting from this point taking $|S|$ continuous 
increasing curves to the vertices of the subsimplex; monotonicity of such an $S$-path means that these curves do not increase in any of the other coordinates.

Observe that if $|S|=2$ in Definition 1, then an elementary $S$-path $\left(p,\left(f^{i}\right)_{i \in S}\right)$ is uniquely determined by its starting point $p$.

\section{Definition 2.}

(a) An $N$-path $\Pi$ assigns to every $S \subseteq N$ with $|S| \geq 2$ a collection of elementary $S$-paths, described recursively as follows:

(0) $\left(p,\left(f^{i}\right)_{i \in N}\right)$ is an elementary $N$-path.

(1) For all $i_{1} \in N$ and all $t_{i_{1}} \in\left[p_{i_{1}}, 1\right]$,

$$
\left(f^{i_{1}}\left(t_{i_{1}}\right),\left(f^{i_{1}, t_{i_{1}} ; j}\right)_{j \in N \backslash\left\{i_{1}\right\}}\right)
$$

is an elementary $N \backslash\left\{i_{1}\right\}$-path.

(k) Whenever $n \geq 4$, for $2 \leq k \leq n-2$, all distinct $i_{1}, \ldots, i_{k} \in N$, and all

$$
\begin{gathered}
t_{i_{k}} \in\left[f^{i_{1}, t_{i_{1}} ; \ldots ; i_{k-2}, t_{i_{k-2}} ; i_{k-1}}\left(t_{i_{k-1}}\right)_{i_{k}}, 1-\sum_{\ell=1}^{k-1} t_{i_{\ell}}\right] \\
\left(f^{i_{1}, t_{i_{1}} ; \ldots ; i_{k-1}, t_{i_{k-1}} ; i_{k}}\left(t_{i_{k}}\right),\left(f^{i_{1}, t_{i_{1}} ; \ldots ; i_{k}, t_{i_{k}} ; j}\right)_{j \in N \backslash\left\{i_{1}, \ldots, i_{k}\right\}}\right)
\end{gathered}
$$

is an elementary $N \backslash\left\{i_{1}, \ldots, i_{k}\right\}$-path.

(b) The $N$-path $\Pi$ is consistent if, whenever $n \geq 4$, for all $2 \leq k \leq n-2$, all distinct $i_{1}, \ldots, i_{k}$ and all permutations $\pi$ of $i_{1}, \ldots, i_{k}$, if

$$
t_{\pi\left(i_{\ell}\right)} \in\left[f^{\pi\left(i_{1}\right), t_{\pi\left(i_{1}\right)} ; \ldots ; \pi\left(i_{\ell-2}\right), t_{\pi\left(i_{\ell-2}\right)} ; \pi\left(i_{\ell-1}\right)}\left(t_{\pi\left(i_{\ell-1}\right)}\right)_{\pi\left(i_{\ell}\right)}, 1-\sum_{j=1}^{\ell-1} t_{\pi\left(i_{j}\right)}\right]
$$

for every $\ell=2, \ldots, k$, then

$$
f^{i_{1}, t_{i_{1}} ; \ldots ; i_{k-1}, t_{i_{k-1}} ; i_{k}}\left(t_{i_{k}}\right)=f^{\pi\left(i_{1}\right), t_{\pi\left(i_{1}\right)} ; \ldots ; \pi\left(i_{k-1}\right), t_{\pi\left(i_{k-1}\right)} ; \pi\left(i_{k}\right)}\left(t_{\pi\left(i_{k}\right)}\right)
$$

for all $t_{i_{1}}, \ldots, t_{i_{k}}$ in the respective domains.

(c) The $N$-path $\Pi$ is continuous if for the functions $f^{i_{1}, t_{i_{1}} ; \ldots ; i_{k}, t_{i} ; j}$ in (1) we have that, for all sequences $\left\langle\left(t_{i_{1}}^{q}, \ldots, t_{i_{k}}^{q}\right)\right\rangle_{q \in \mathbb{N}}$ and all $\left(t_{i_{1}}^{\prime}, \ldots, t_{i_{k}}^{\prime}\right)$,

$$
\lim _{q \rightarrow \infty}\left(t_{i_{1}}^{q}, \ldots, t_{i_{k}}^{q}\right)=\left(t_{i_{1}}^{\prime}, \ldots, t_{i_{k}}^{\prime}\right) \Rightarrow \lim _{q \rightarrow \infty} f^{i_{1}, t_{i_{1}}^{q} ; \ldots ; i_{k}, t_{i_{k}}^{q} ; j}\left(t_{j}\right)=f^{i_{1}, t_{i_{1}}^{\prime} ; \ldots ; i_{k}, t_{i_{k}}^{\prime} ; j}\left(t_{j}\right)
$$

for every $t_{j}$ in the respective domains.

(d) The $N$-path $\Pi$ is monotonic if each elementary $S$-path belonging to it is monotonic. 
Thus, an $N$-path starts with an elementary $N$-path, consisting of $n$ continuous curves from a point $p$ to each of the vertices. Next, for each curve $f^{i}$ and each point $f^{i}\left(t_{i}\right)$ on that curve, we have an elementary $N \backslash\{i\}$ path in the subsimplex obtained by fixing the $i^{\text {th }}$ coordinate at $t_{i}$. This process is repeated until we arrive at (uniquely determined) elementary $S$-paths for $|S|=2$.

Consistency of an $N$-path means that if we move a number of steps along the $N$-path and reach a certain point, then we reach the same point if we permute the steps in this movement; condition (2) restricts this requirement to well-defined movements.

Continuity requires that, in addition to the implicit property that all curves in elementary paths are continuous, all curves behave continuously with respect to changes in starting points.

Monotonicity means that all elementary paths in the requisite $N$-paths are monotonic.

We will now define a choice function based on a consistent, continuous, and monotonic $N$-path.

\section{Definition 3.}

Let $\Pi$ be an $N$-path as in Definition 2 , and let $\Pi$ be consistent, continuous, and monotonic. The choice function $C^{\Pi}$ is determined via the following algorithm. Let $\alpha \in A$.

(0) If $p^{0}:=p \geq \alpha$ then $C^{\Pi}(\alpha)=p^{0}$.

Otherwise, go to Step 1.

(1) Let $N^{1}=\left\{i \in N \mid \alpha_{i}>p_{i}^{0}\right\}$ and let $i_{1}, \ldots, i_{\ell_{1}}$ be an ordering of $N^{1}$. If $p^{1}:=f^{i_{1}, \alpha_{i_{1}} ; \ldots ; i_{\ell_{1}-1}, \alpha_{i_{\ell_{1}-1 ;} ; \ell_{1}}}\left(\alpha_{i_{\ell_{1}}}\right) \geq \alpha$, then $C^{\Pi}(\alpha)=p^{1}$.

Otherwise, go to Step 2.

(2) Let $N^{2}=\left\{i \in N \mid \alpha_{i}>p_{i}^{1}\right\}$ and let $i_{\ell_{1}+1}, \ldots, i_{\ell_{2}}$ be an ordering of $N^{2}$. If $p^{2}:=f^{i_{1}, \alpha_{i_{1}} ; \ldots ; i_{\ell_{2}-1}, \alpha_{i_{\ell_{2}-1 ;} ; \ell_{2}}}\left(\alpha_{i_{\ell_{2}}}\right) \geq \alpha$, then $C^{\Pi}(\alpha)=p^{2}$.

Otherwise, go to Step 3.

(k) Let $N^{k}=\left\{i \in N \mid \alpha_{i}>p_{i}^{k-1}\right\}$ and let $i_{\ell_{k-1}+1}, \ldots, i_{\ell_{k}}$ be an ordering of $N^{k}$. If $p^{k}:=f^{i_{1}, \alpha_{i_{1}} ; \ldots ; i_{\ell_{k}-1}, \alpha_{i_{\ell_{k}}-1 ; \ell_{\ell_{k}}}}\left(\alpha_{i_{\ell_{k}}}\right) \geq \alpha$, then $C^{\Pi}(\alpha)=p^{k}$.

Otherwise, go to Step $k+1$.

In words, in order to determine the outcome in a particular division problem $\Delta(\alpha)$, we start from the solution $p$ of the problem $\Delta(0)$ and successively increase, along the curves in the elementary $N$-path, the shares in all coordinates (individuals) for which such an increase is possible. If we reach the subsimplex $\Delta(\alpha)$, the algorithm terminates and the choice is determined. Otherwise, we continue with the next set of coordinates along curves in the associated elementary paths and continue until we reach $\Delta(\alpha)$. The consistency requirement guarantees that, in the requisite consecutive steps, the order in which we increase the coordinates does not matter. The proof of the following theorem formalizes this. For the case $n=3$, the following section provides some diagrammatic illustrations of the process involved in this construction. 
Theorem 1. Let $\Pi$ be a consistent, continuous, and monotonic $N$-path. Then $C^{\Pi}$ is well-defined, continuous, monotonic, and independent of irrelevant alternatives.

Proof. Clearly, since the algorithm defining $C^{\Pi}$ follows a sequence of functions in elementary paths, it terminates at or before Step $n-1$ for every $\alpha \in A$. If the algorithm terminates at Step 1, then by consistency of $\Pi$ the ordering of the elements of $N^{1}$ does not matter; moreover, monotonicity of each of the curves used in Step 1 ensures that Step 1 is well-defined for any ordering. Similarly, if the algorithm terminates at Step 2, then the ordering of the elements of $N^{2}$ does not matter, etc. Hence, $C^{\Pi}$ is well-defined.

Continuity of $C^{\Pi}$ follows from continuity of $\Pi$ and continuity of the functions in every elementary $S$-path in $\Pi$.

To prove monotonicity of $C^{\Pi}$, let $\alpha \in A, i \in N$, and $\alpha_{i}^{\prime} \in(0,1)$ be such that $\alpha_{i}^{\prime}>\alpha_{i}$ and $\left(\alpha_{-i}, \alpha_{i}^{\prime}\right) \in A$, and let $j \in N$ be such that $C^{\Pi}(\alpha)_{j}>\alpha_{j}$. Also let $C^{\Pi}(\alpha)_{i}=\alpha_{i}$. We have to show that $C^{\Pi}\left(\alpha_{-i}, \alpha_{i}^{\prime}\right)_{j} \leq C^{\Pi}(\alpha)_{j}$. Let $N_{\alpha}$ and $N_{\alpha^{\prime}}$ denote the unions of the sets $N_{\alpha}^{1}, N_{\alpha}^{2}, \ldots$ and $N_{\alpha^{\prime}}^{1}, N_{\alpha^{\prime}}^{2}, \ldots$ in the algorithm for $C^{\Pi}(\alpha)$ and $C^{\Pi}\left(\alpha^{\prime}\right)$, respectively. If $i \notin N_{\alpha^{\prime}}$, then $i \notin N_{\alpha}$ which, in turn, implies $C^{\Pi}(\alpha)=C^{\Pi}\left(\alpha^{\prime}\right)$ so that in particular $C^{\Pi}\left(\alpha_{-i}, \alpha_{i}^{\prime}\right)_{j} \leq C^{\Pi}(\alpha)_{j}$. If $i \in N_{\alpha^{\prime}}$, then if (say) $C^{\Pi}\left(\alpha^{\prime}\right)=f^{i_{1}, \alpha_{i_{1}}^{\prime} ; \ldots ; i_{\ell_{k}-1}, \alpha_{i_{\ell_{k}}-1^{;} ; \ell_{k}}^{\prime}}\left(\alpha_{i_{\ell_{k}}}^{\prime}\right)$, we may assume that $i=i_{\ell_{k}}$. Then either $C^{\Pi}(\alpha)=f^{i_{1}, \alpha_{i_{1}} ; \ldots ; i_{\ell_{k}-1}}\left(\alpha_{i_{\ell_{k}-1}}\right)$ (if the $i$-th coordinate of this point is equal to $\alpha_{i}$ ) or $C^{\Pi}(\alpha)=f^{i_{1}, \alpha_{i_{1}} ; \ldots ; i_{\ell_{k}-1}, \alpha_{i_{\ell_{k}}-1 ; \ell_{\ell_{k}}}}\left(\alpha_{i_{\ell_{k}}}\right)$ (if the $i$-th coordinate of the previous point was strictly smaller than $\left.\alpha_{i}\right)$. In either case, $C^{\Pi}\left(\alpha_{-i}, \alpha_{i}^{\prime}\right)_{j} \leq C^{\Pi}(\alpha)_{j}$ follows from the monotonicity of the function $f^{i_{1}, \alpha_{i_{1}} ; \ldots ; i_{\ell_{k}-1}, \alpha_{i_{\ell_{k}}-1 ; \ell_{k}}}$.

In order to show that $C^{\Pi}$ is independent of irrelevant alternatives, let $\alpha, \beta \in A$ with $C^{\Pi}(\alpha) \geq \beta \geq \alpha$. We wish to show that $C^{\Pi}(\alpha)=C^{\Pi}(\beta)$. Clearly, if $p \geq \alpha$, then $p=C^{\Pi}(\alpha) \geq \beta$, hence $C^{\Pi}(\beta)=p=C^{\Pi}(\alpha)$. Now denote the sets $N^{1}, N^{2}, \ldots$ and the points $p^{1}, p^{2}, \ldots$ in the algorithm applied to $\alpha$ and $\beta$ by $N_{\alpha}^{1}, N_{\alpha}^{2}, \ldots ; N_{\beta}^{1}, N_{\beta}^{2}, \ldots$, and $p_{\alpha}^{1}, p_{\alpha}^{2}, \ldots ; p_{\beta}^{1}, p_{\beta}^{2}, \ldots$, respectively. Suppose $C^{\Pi}(\alpha)$ is determined in Step $k \geq 1$. Then, for each $i \in N_{\alpha}^{1} \cup \ldots \cup N_{\alpha}^{k}$, we have $\alpha_{i}=C^{\Pi}(\alpha)_{i} \geq \beta_{i} \geq \alpha_{i}$ so that $\alpha_{i}=\beta_{i}$. This implies $N_{\alpha}^{1} \subseteq N_{\beta}^{1}$. Suppose that $j \notin N_{\alpha}^{1} \cup \ldots \cup N_{\alpha}^{k}$ but $j \in N_{\beta}^{1}$. Then $\beta_{j}>p_{j} \geq \alpha_{j}$ since, in particular, $j \notin N_{\alpha}^{1}$. Moreover, the monotonicity of $\Pi$ implies that $p_{j} \geq C^{\Pi}(\alpha)_{j}$. Hence $p_{j} \geq \beta_{j}$, a contradiction. Therefore, $N_{\beta}^{1} \subseteq N_{\alpha}^{1} \cup \ldots \cup N_{\alpha}^{k}$, but then we have $N_{\beta}^{1}=N_{\alpha}^{1}$ since $\alpha_{i}=\beta_{i}$ for all $i \in N_{\alpha}^{1} \cup \ldots \cup N_{\alpha}^{k}$. Next, suppose again that $j \notin N_{\alpha}^{1} \cup \ldots \cup N_{\alpha}^{k}$, and assume that $N_{\beta}^{r}=N_{\alpha}^{r}$ for all $r=1, \ldots, \ell-1$, where $\ell \in\{2, \ldots, k\}$. Suppose that $j \in N_{\beta}^{\ell}$. Then $\beta_{j}>\left(p_{\beta}^{\ell-1}\right)_{j}=\left(p_{\alpha}^{\ell-1}\right)_{j} \geq \alpha_{j}$, and by monotonicity $\left(p_{\alpha}^{\ell-1}\right)_{j} \geq C^{\Pi}(\alpha)_{j}$. Hence $\left(p_{\beta}^{\ell-1}\right)_{j}=\left(p_{\alpha}^{\ell-1}\right)_{j} \geq C^{\Pi}(\alpha)_{j} \geq \beta_{j}$, a contradiction. This implies $N_{\beta}^{\ell} \subseteq N_{\alpha}^{1} \cup \ldots \cup N_{\alpha}^{k}$, and thus again $N_{\beta}^{\ell}=N_{\alpha}^{\ell}$. Since $C^{\Pi}(\alpha) \geq \beta$, we conclude that $C^{\Pi}(\beta)=C^{\Pi}(\alpha)$.

The next result establishes the converse of Theorem 1.

Theorem 2. Let $C$ be a choice function satisfying independence of irrelevant alternatives, continuity, and monotonicity. Then there exists a consistent, continuous, and monotonic $N$-path $\Pi$ such that $C=C^{\Pi}$.

Proof. We iteratively construct an $N$-path $\Pi$ as follows. 
(0) Let $p=C(0, \ldots, 0)$ and for every $i \in N$ let $f^{i}$ be defined by $f^{i}\left(t_{i}\right)=C\left(t_{i} e^{i}\right)$ for every $t_{i} \in\left[p_{i}, 1\right]$. Then $f^{i}\left(t_{i}\right)=t_{i}$ by Lemma 1 . Moreover, the functions $f^{i}$ are continuous and monotonic by the continuity and monotonicity of $C$. Hence, $\left(p,\left(f^{i}\right)_{i \in N}\right)$ is an elementary $N$-path which is monotonic.

(1) For all distinct $i, j \in N$ and $t_{i} \in\left[p_{i}, 1\right]$, let $f^{i, t_{i} ; j}$ be defined by $f^{i, t_{i} ; j}\left(t_{j}\right)=C\left(t_{i} e^{i}+t_{j} e^{j}\right)$ for every $t_{j} \in\left[f^{i}\left(t_{i}\right)_{j}, 1-t_{i}\right]$. Then $f^{i, t_{i} ; j}\left(t_{j}\right)=t_{j}$ by Lemma 1 . Moreover, the functions $f^{i, t_{i} ; j}$ are continuous and monotonic by the continuity and monotonicity of $C$. Hence, the pair $\left(f^{i}\left(t_{i}\right),\left(f^{i, t_{i} ; j}\right)_{j \in N \backslash\{i\}}\right)$ is an elementary $N$-path which is monotonic.

(k) For $2 \leq k \leq n-2$, all distinct $i_{1}, \ldots, i_{k} \in N$, and $t_{i_{1}}, \ldots, t_{i_{k-1}}$ with $\sum_{j=1}^{k-1} t_{i_{j}}<$ 1 , let, for every $\left.t_{i_{k}} \in\left[f^{i_{1}, t_{1} ; \ldots ; i_{k-1}}\left(t_{i_{k-1}}\right)_{i_{k}}\right), 1-\sum_{j=1}^{k-1} t_{i_{j}}\right], f^{i_{1}, t_{i_{1}} ; \ldots ; i_{k-1}, t_{i_{k-1}} ; i_{k}}\left(t_{i_{k}}\right)=$ $C\left(\sum_{j=1}^{k} t_{i_{j}} e^{i_{j}}\right)$. Then $f^{i_{1}, t_{i_{1}} ; \ldots ; i_{k-1}, t_{i_{k-1}} ; i_{k}}\left(t_{i_{k}}\right)=t_{i_{k}}$ by Lemma 1 . Moreover, the functions $f^{i_{1}, t_{i_{1}} ; \ldots ; i_{k-1}, t_{i_{k-1}} ; i_{k}}$ are continuous and monotonic by the continuity and monotonicity of $C$. Hence, $\left.\left(f^{i_{1}, t_{1} ; \ldots ; i_{k-1}}\left(t_{i_{k-1}}\right), f^{i_{1}, t_{1} ; \ldots ; i_{k-1}, t_{i_{k-1}} ; j}\right)_{j \in N \backslash\left\{i_{1}, \ldots, i_{k-1}\right\}}\right)$ is an elementary $N \backslash\left\{i_{1}, \ldots, i_{k-1}\right\}$-path which is monotonic.

The consistency of $\Pi$ follows by construction, and the continuity of $\Pi$ follows by the continuity of $C$. Finally, we have $C(\alpha)=C^{\Pi}(\alpha)$ for all $\alpha \in A$ by the construction of $\Pi$.

\section{The case $n=3$}

In the case $n=3$, an $N$-path is completely described by its elementary $N$-path in Step 0 of Definition 2, since for distinct $i, j \in N$ the elementary $\{i, j\}$-paths in Step 1 are uniquely determined by (ii) in Definition 1 . This makes it is also easy to see that $N$-paths are continuous and consistent by definition. Thus, for $N=\{1,2,3\}$ an $N$-path can be identified with an elementary $N$-path $\Pi=\left(p,\left(f^{i}\right)_{i \in N}\right)$. We weaken monotonicity of an $N$-path to the following condition. The $N$-path $\Pi=\left(p,\left(f^{i}\right)_{i \in N}\right)$ is weakly monotonic if for all $i, j \in N$ and all $t_{i} \in\left[p_{i}, 1\right]$ and $t_{j} \in\left[p_{j}, 1\right]$ such that $t_{i}+t_{j}<1$,

$$
\left[f^{i}\left(t_{i}\right)_{j} \geq t_{j} \text { and } f^{j}\left(t_{j}\right)_{i} \geq t_{i}\right] \Rightarrow f^{i}\left(t_{i}\right)=f^{j}\left(t_{j}\right) .
$$

For a monotonic $N$-path the premise in (3) is only satisfied for $t_{i}=p_{i}$ and $t_{j}=p_{j}$, in which case $f^{i}\left(t_{i}\right)=f^{j}\left(t_{j}\right)=p$, hence a monotonic $N$-path satisfies (3).

Now suppose that the choice function $C$ is independent of irrelevant alternatives and continuous. As in the proof of Theorem 2, let $p=C(0,0,0)$ and for each $i \in N$ and $t_{i} \in\left[p_{i}, 1\right]$ let $f^{i}\left(t_{i}\right)=C\left(t_{i} e^{i}\right)$. Then, by independence of irrelevant alternatives and continuity (and, in particular, Lemma 1$), \Pi=\left(p,\left(f^{i}\right)_{i \in N}\right)$ is an (elementary) $N$-path such that $C(\alpha)=C^{\Pi}(\alpha)$ for every $\alpha \in A$. Moreover, suppose that the premise of (3) holds. Then $f^{i}\left(t_{i}\right), f^{j}\left(t_{j}\right) \in \Delta\left(t_{i} e^{i}+t_{j} e^{j}\right)$; since $\Delta\left(t_{i} e^{i}+t_{j} e^{j}\right) \subseteq \Delta\left(t_{i} e^{i}\right) \cap \Delta\left(t_{j} e^{j}\right)$, independence of irrelevant alternatives of $C$ implies $f^{i}\left(t_{i}\right)=f^{j}\left(t_{j}\right)$. Hence (3) holds.

Conversely, for an (elementary) $N$-path $\Pi=\left(p,\left(f^{i}\right)_{i \in N}\right)$ satisfying weak monotonicity we construct a choice function $C^{\Pi}$ analogous to Definition 3 - this construction now greatly 
simplifies as follows. Let $\alpha \in A$ and define

$$
N_{\alpha>p}=\left\{i \in N \mid \alpha_{i}>p_{i}\right\} .
$$

Then

(a) if $N_{\alpha>p}=\emptyset$, then $C^{\Pi}(\alpha)=p$;

(b) if $N_{\alpha>p} \neq \emptyset$, then $C^{\Pi}(\alpha)=\arg \min _{x \geq \alpha}\left\{\left\|x-f^{i}(\alpha)\right\|\right.$ for some $\left.i \in N_{\alpha>p}\right\}$.

By (3), $C^{\Pi}(\alpha)$ in (b) is well-defined. It is easy to verify that $C^{\Pi}$ is independent of irrelevant alternatives and continuous. Summarizing, we have the following theorem.

Theorem 3. Let $n=3$. A choice function $C$ satisfies independence of irrelevant alternatives and continuity if and only if there is a weakly monotonic (elementary) $N$-path $\Pi=\left(p,\left(f^{i}\right)_{i \in N}\right)$ such that $C=C^{\Pi}$.

For $n=3$, an example of an $N$-path $\Pi=(p, f)=\left(p,\left(f^{i}\right)_{i \in N}\right)$ and the associated choice function $C^{\Pi}$ is presented in the following example. Figures 2 to 5 provide illustrations for different choices of $\left(f^{i}\right)_{i \in N}$ and different values of the parameter $\alpha=\left(\alpha_{1}, \alpha_{2}, \alpha_{3}\right)$.

Example 1. Throughout the example, the point $p$ is given $p=\left(\frac{3}{8}, \frac{1}{8}, \frac{1}{2}\right)$ and in each of Figures 2 to 4 , the set $\Delta(\alpha)$ is indicated by the shaded area.

(a) In Figure 2, we let $\alpha=(0,0,0)$. The three paths leading from $p$ to each of the three vertices of $\Delta(0,0,0)$ are associated with the functions $f^{1}, f^{2}$, and $f^{3}$. We obtain $C^{\Pi}(\alpha)=p$ by definition.

(b) In Figure 3, we illustrate the choice for $\alpha=\left(0,0, \frac{1}{4}\right)$. Because $N_{\alpha>p}=\emptyset$, part (a) of the definition of an $N$-path choice function implies that $C^{\Pi}(\alpha)=p=\left(\frac{3}{8}, \frac{1}{8}, \frac{1}{2}\right)$.

(c) Figure 4 depicts the choice for $\alpha=\left(\frac{1}{4}, \frac{3}{8}, \frac{1}{8}\right)$. We have $p_{1}=\frac{3}{8} \geq \frac{1}{4}=\alpha_{1}, p_{2}=\frac{1}{8}<$ $\frac{3}{8}=\alpha_{2}$ and $p_{3}=\frac{1}{2} \geq \frac{1}{8}=\alpha_{3}$ and, thus, $N_{\alpha>p}=\{2\}$. Furthermore, $f^{2}\left(\alpha_{2}\right)=\left(\frac{1}{4}, \frac{3}{8}, \frac{3}{8}\right)=$ $y \geq\left(\frac{1}{4}, \frac{3}{8}, \frac{1}{8}\right)=\alpha$. According to part (b) of our definition, we obtain $C^{\Pi}(\alpha)=\left(\frac{1}{4}, \frac{3}{8}, \frac{3}{8}\right)$.

(d) Finally, Figure 5 illustrates the case $\alpha=\left(\frac{1}{2}, \frac{3}{8}, 0\right)$. According to our definition, we obtain $C^{\Pi}(\alpha)=\left(\frac{1}{2}, \frac{3}{8}, \frac{1}{8}\right)=y$. Note that we have $N_{\alpha>p}=\{1,2\}, C_{2}(\alpha)=y_{2}=\frac{3}{8}=\alpha_{2}$, and $C_{1}(\alpha)=y_{1}=\frac{1}{2}=\alpha_{1}$. 


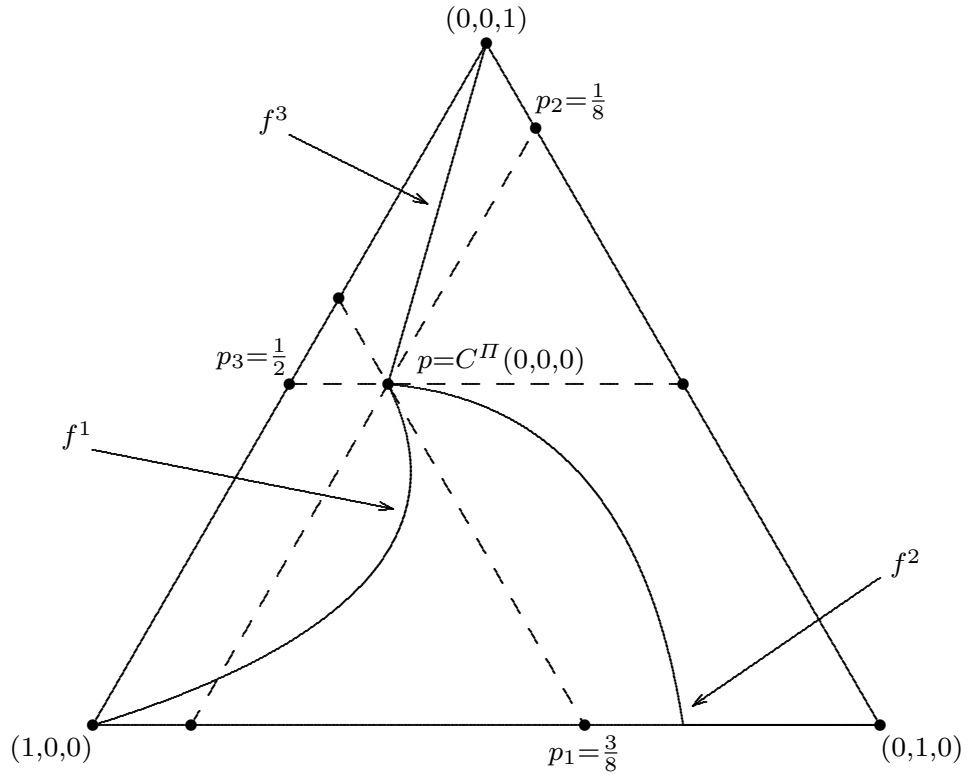

Figure 2: Example 1, part (a).

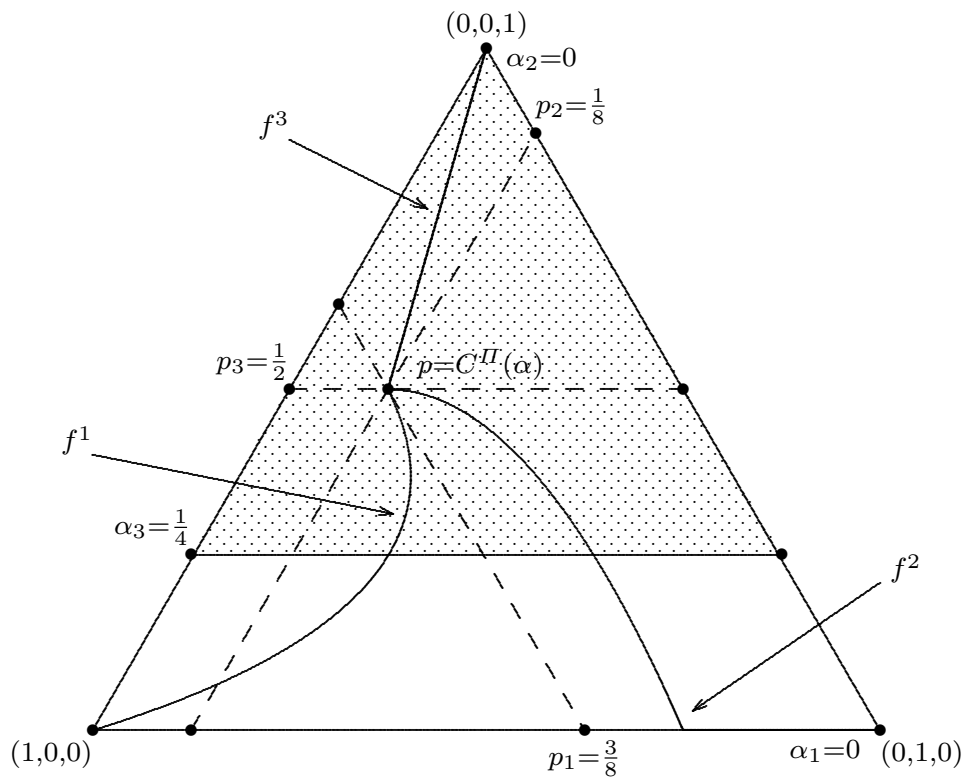

Figure 3: Example 1, part (b). 


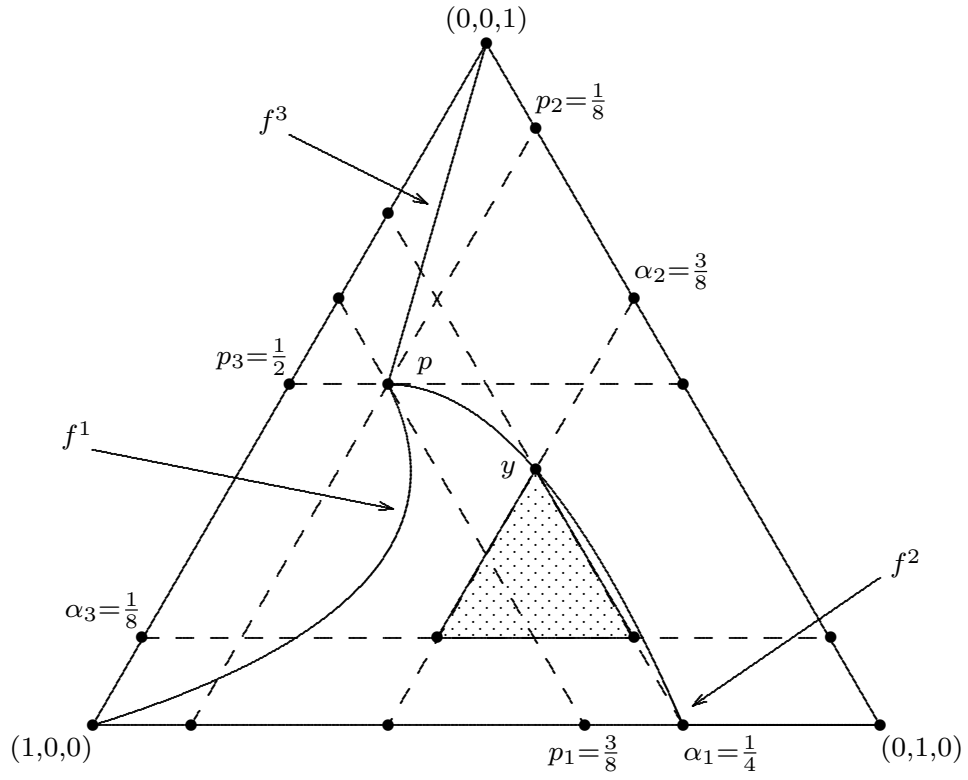

Figure 4: Example 1, part (c).

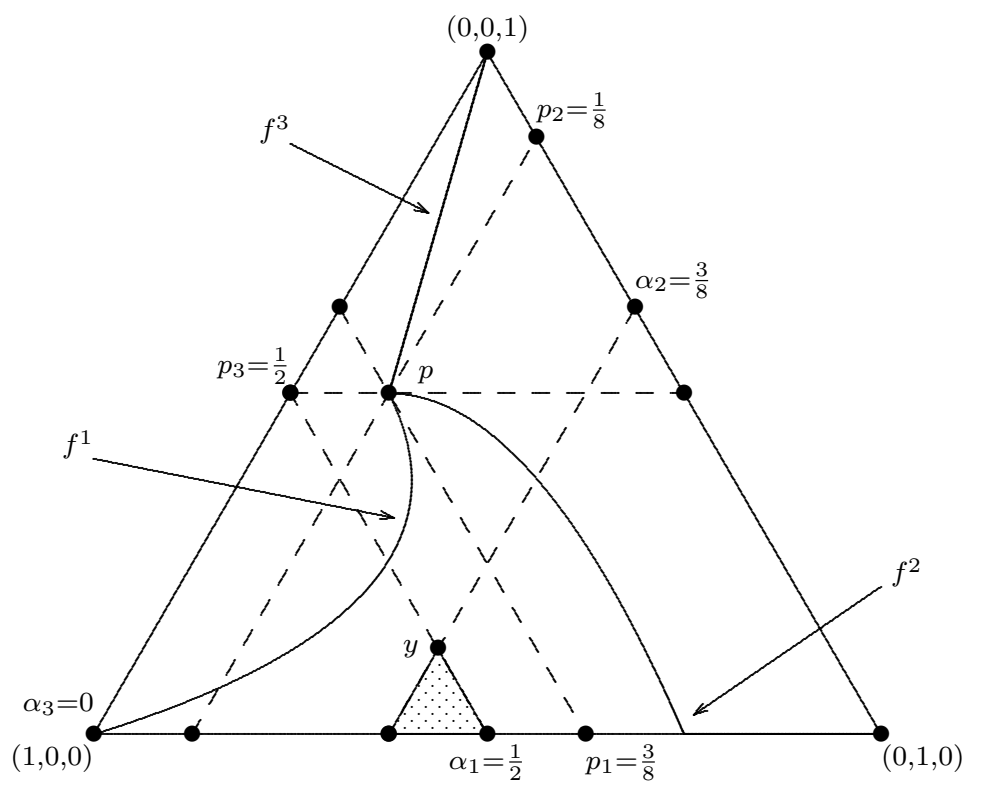

Figure 5: Example 1, part (d). 


\section{Rationalizability in the case $n=3$}

The notion of rationalizability is a fundamental issue in the theory of individual and collective choice. In our context, a choice function $C$ is rationalizable if there exists an ordering $R$ on $\Delta(0, \ldots, 0)$ such that, for all $\alpha \in A$ and for all $x \in \Delta(\alpha)$,

$$
C(\alpha)=x \Leftrightarrow x R y \text { for all } y \in \Delta(\alpha) .
$$

The direct revealed preference relation $R^{C}$ corresponding to the choice function $C$ is defined by letting, for all $x, y \in \Delta(0, \ldots, 0), x R^{C} y$ if there exists $\alpha \in A$ such that $C(\alpha)=x$ and $y \geq \alpha$. A revealed-preference cycle in $C$ of length $K \in \mathbb{N} \backslash\{1\}$ is a set of distinct alternatives $\left\{x^{1}, \ldots, x^{K}\right\}$ such that $x^{k} R^{C} x^{k+1}$ for all $k \in\{1, \ldots, K\}$, where $x^{K+1}=x^{1}$. The following axioms are well-established in the theory of rational choice.

Weak axiom of revealed preference. There is no revealed-preference cycle of length two in $C$.

Strong axiom of revealed preference. For all $K \in \mathbb{N} \backslash\{1\}$, there is no revealedpreference cycle of length $K$ in $C$.

It is well-known that, because our choice function is single-valued, rationalizability is equivalent to the strong axiom of revealed preference. The strong axiom of revealed preference implies the weak axiom which, in turn, implies independence of irrelevant alternatives. This is true for a choice function with an arbitrary domain. Because the domain of our choice function is closed under intersection, the weak axiom of revealed preference is equivalent to the independence property.

We now illustrate that, on our domain, independence of irrelevant alternatives (or, equivalently, the weak axiom of revealed preference) is not sufficient for rationalizability in the case $n=3$. Moreover, for any $K \in \mathbb{N}$, ruling out the existence of revealed-preference cycles of any length less than $K+1$ is not sufficient to rule out cycles of length $K+1$. This is established in the following theorem. For that case, we denote the left, right and top vertices of a simplex $\Delta(\alpha)$ by $\ell(\alpha), r(\alpha)$, and $t(\alpha)$, hence $\ell(\alpha)=\left(1-\alpha_{2}-\alpha_{3}, \alpha_{2}, \alpha_{3}\right)$, $r(\alpha)=\left(\alpha_{1}, 1-\alpha_{1}-\alpha_{3}, \alpha_{3}\right)$, and $t(\alpha)=\left(\alpha_{1}, \alpha_{2}, 1-\alpha_{1}-\alpha_{2}\right)$.

Theorem 4. Let $n=3$. For each $K \in \mathbb{N}$, there exists a choice function $C^{K+1}$ on the simplex domain such that there is a revealed-preference cycle of length $K+1$ in $C^{K+1}$ and no revealed-preference cycle of length less than $K+1$ in $C^{K+1}$.

Proof. For the case $K=1$, consider the choice function $C^{2}$ defined by letting, for all $\alpha \in A$,

$$
C^{2}(\alpha)= \begin{cases}\ell(\alpha) & \text { if } \alpha=(0,0,0), \\ r(\alpha) & \text { if } \alpha \neq(0,0,0) .\end{cases}
$$

Then

$$
\begin{array}{r}
C^{2}(0,0,0)=(1,0,0) \text { and }\left(\frac{1}{2}, \frac{1}{2}, 0\right) \geq(0,0,0) ; \\
C^{2}\left(\frac{1}{2}, 0,0\right)=\left(\frac{1}{2}, \frac{1}{2}, 0\right) \text { and }(1,0,0) \geq\left(\frac{1}{2}, 0,0\right)
\end{array}
$$


so that the points $(1,0,0)$ and $\left(\frac{1}{2}, 0,0\right)$ form a cycle of length two (and, trivially, there is no cycle of length less than two).

For the case $K=2$, consider the choice function $C^{3}$ defined by letting, for all $\alpha \in A$,

$$
C^{3}(\alpha)= \begin{cases}\ell(\alpha) & \text { if } \alpha_{3} \geq \frac{1}{2} \\ r(\alpha) & \text { if } \alpha_{1} \geq \frac{1}{2} \\ t(\alpha) & \text { if } \alpha_{2} \geq \frac{1}{2} \\ \arg \min _{x \geq \alpha}\left\|x-\left(\frac{1}{3}, \frac{1}{3}, \frac{1}{3}\right)\right\| & \text { otherwise }\end{cases}
$$

Then

$$
\begin{aligned}
& C^{3}\left(0, \frac{1}{2}, 0\right)=\left(0, \frac{1}{2}, \frac{1}{2}\right) \quad \text { and } \quad\left(\frac{1}{2}, \frac{1}{2}, 0\right) \geq\left(0, \frac{1}{2}, 0\right) \\
& C^{3}\left(\frac{1}{2}, 0,0\right)=\left(\frac{1}{2}, \frac{1}{2}, 0\right) \quad \text { and } \quad\left(\frac{1}{2}, 0, \frac{1}{2}\right) \geq\left(\frac{1}{2}, 0,0\right) \\
& C^{3}\left(0,0, \frac{1}{2}\right)=\left(\frac{1}{2}, 0, \frac{1}{2}\right) \quad \text { and } \quad\left(0, \frac{1}{2}, \frac{1}{2}\right) \geq\left(0,0, \frac{1}{2}\right)
\end{aligned}
$$

so that we have a cycle of length three formed by the points $\left(0, \frac{1}{2}, \frac{1}{2}\right),\left(\frac{1}{2}, \frac{1}{2}, 0\right)$ and $\left(\frac{1}{2}, 0, \frac{1}{2}\right)$ but there is no cycle of length two.

For the case $K=3$, consider the choice function $C^{4}$ defined by letting, for all $\alpha \in A$,

$$
C^{4}(\alpha)= \begin{cases}C_{*}^{4}(\alpha) & \text { if } \alpha_{3} \geq \frac{1}{2} \\ C^{3}(\alpha) & \text { if } \alpha_{3}<\frac{1}{2}\end{cases}
$$

where $C_{*}^{4}:\left\{\alpha \in A \mid \alpha_{3} \geq \frac{1}{2}\right\} \rightarrow \Delta(0,0,0)$ is defined by

$$
C_{*}^{4}(\alpha)= \begin{cases}\ell(\alpha) & \text { if } \alpha_{1} \geq \frac{1}{4} \text { or } \alpha_{2} \geq \frac{1}{4}, \\ \arg \min _{x \geq \alpha}\left\|x-\left(\frac{1}{8}, \frac{1}{8}, \frac{3}{4}\right)\right\| & \text { otherwise. }\end{cases}
$$

Now we obtain

$$
\begin{aligned}
C^{4}\left(0, \frac{1}{2}, 0\right) & =\left(0, \frac{1}{2}, \frac{1}{2}\right) \text { and }\left(\frac{1}{2}, \frac{1}{2}, 0\right) \geq\left(0, \frac{1}{2}, 0\right) ; \\
C^{4}\left(\frac{1}{2}, 0,0\right) & =\left(\frac{1}{2}, \frac{1}{2}, 0\right) \text { and }\left(\frac{1}{2}, 0, \frac{1}{2}\right) \geq\left(\frac{1}{2}, 0,0\right) ; \\
C^{4}\left(\frac{1}{4}, 0, \frac{1}{2}\right) & =\left(\frac{1}{2}, 0, \frac{1}{2}\right) \text { and }\left(\frac{1}{4}, \frac{1}{4}, \frac{1}{2}\right) \geq\left(\frac{1}{4}, 0, \frac{1}{2}\right) ; \\
C^{4}\left(0, \frac{1}{4}, \frac{1}{2}\right) & =\left(\frac{1}{4}, \frac{1}{4}, \frac{1}{2}\right) \text { and }\left(0, \frac{1}{2}, \frac{1}{2}\right) \geq\left(0, \frac{1}{4}, \frac{1}{2}\right)
\end{aligned}
$$

so that $C^{4}$ has a cycle of length four formed by the points $\left(0, \frac{1}{2}, \frac{1}{2}\right),\left(\frac{1}{2}, \frac{1}{2}, 0\right),\left(\frac{1}{2}, 0, \frac{1}{2}\right)$, $\left(\frac{1}{4}, \frac{1}{4}, \frac{1}{2}\right)$ but no cycles of smaller length.

This last choice function can be generalized to all $K \geq 3$ as follows. Consider the choice function $C^{K+1}$ defined by letting, for all $\alpha \in A$,

$$
C^{K+1}(\alpha)= \begin{cases}C_{*}^{K+1}(\alpha) & \text { if } \alpha_{3} \geq \frac{1}{2} \text { and } \alpha_{2} \geq \frac{2^{K-3}-1}{2^{K-2}} \\ C^{K}(\alpha) & \text { otherwise }\end{cases}
$$


where $C_{*}^{K+1}:\left\{\alpha \in A \mid \alpha_{3} \geq \frac{1}{2}\right.$ and $\left.\alpha_{2} \geq \frac{2^{K-3}-1}{2^{K-2}}\right\} \rightarrow \Delta(0,0,0)$ is defined by $C_{*}^{K+1}(\alpha)= \begin{cases}\ell(\alpha) & \text { if } \alpha_{1} \geq \frac{1}{2^{K-1}} \text { or } \alpha_{2} \geq \frac{2^{K-2}-1}{2^{K-1}}, \\ \arg \min _{x \geq \alpha}\left\|x-\left(\frac{1}{2^{K}}, \frac{2^{K}-2^{K-1}-3}{2^{K}}, \frac{2^{K-1}+2}{2^{K}}\right)\right\| & \text { otherwise. }\end{cases}$

We have

$$
\begin{gathered}
C^{K+1}\left(0, \frac{1}{2}, 0\right)=\left(0, \frac{1}{2}, \frac{1}{2}\right) \quad \text { and }\left(\frac{1}{2}, \frac{1}{2}, 0\right) \geq\left(0, \frac{1}{2}, 0\right) ; \\
C^{K+1}\left(\frac{1}{2}, 0,0\right)=\left(\frac{1}{2}, \frac{1}{2}, 0\right) \quad \text { and } \quad\left(\frac{1}{2}, 0, \frac{1}{2}\right) \geq\left(\frac{1}{2}, 0,0\right) ; \\
C^{K+1}\left(\frac{1}{4}, 0, \frac{1}{2}\right)=\left(\frac{1}{2}, 0, \frac{1}{2}\right) \quad \text { and } \quad \ldots \geq\left(\frac{1}{4}, 0, \frac{1}{2}\right) ; \\
C^{K+1}\left(\frac{1}{2^{K-1}}, \frac{2^{K-3}-1}{2^{K-2}}, \frac{1}{2}\right)=\left(\frac{1}{2^{K-2}}, \frac{2^{K-3}-1}{2^{K-2}}, \frac{1}{2}\right) \quad \text { and } \quad\left(\frac{1}{2^{K-1}}, \frac{2^{K-2}-1}{2^{K-1}}, \frac{1}{2}\right) \\
C^{K+1}\left(0, \frac{2^{K-2}-1}{2^{K-1}}, \frac{1}{2}\right)=\left(\frac{1}{2^{K-1}}, \frac{2^{K-2}-1}{2^{K-1}}, \frac{1}{2}\right) \quad \text { and } \quad\left(0, \frac{1}{2}, \frac{1}{2}\right) \geq\left(0, \frac{2^{K-2}-1}{2^{K-1}}, \frac{1}{2}\right)
\end{gathered}
$$

so that $C^{K+1}$ has a cycle of length $K+1$ formed by the points $\left(0, \frac{1}{2}, \frac{1}{2}\right),\left(\frac{1}{2}, \frac{1}{2}, 0\right),\left(\frac{1}{2}, 0, \frac{1}{2}\right)$, $\ldots,\left(\frac{1}{2^{K-2}}, \frac{2^{K-3}-1}{2^{K-2}}, \frac{1}{2}\right),\left(\frac{1}{2^{K-1}}, \frac{2^{K-2}-1}{2^{K-1}}, \frac{1}{2}\right)$, but no cycles of smaller length.

The cases $K=1, K=2, K=3$, and $K=4$ are illustrated in Figures 6 to 9 .

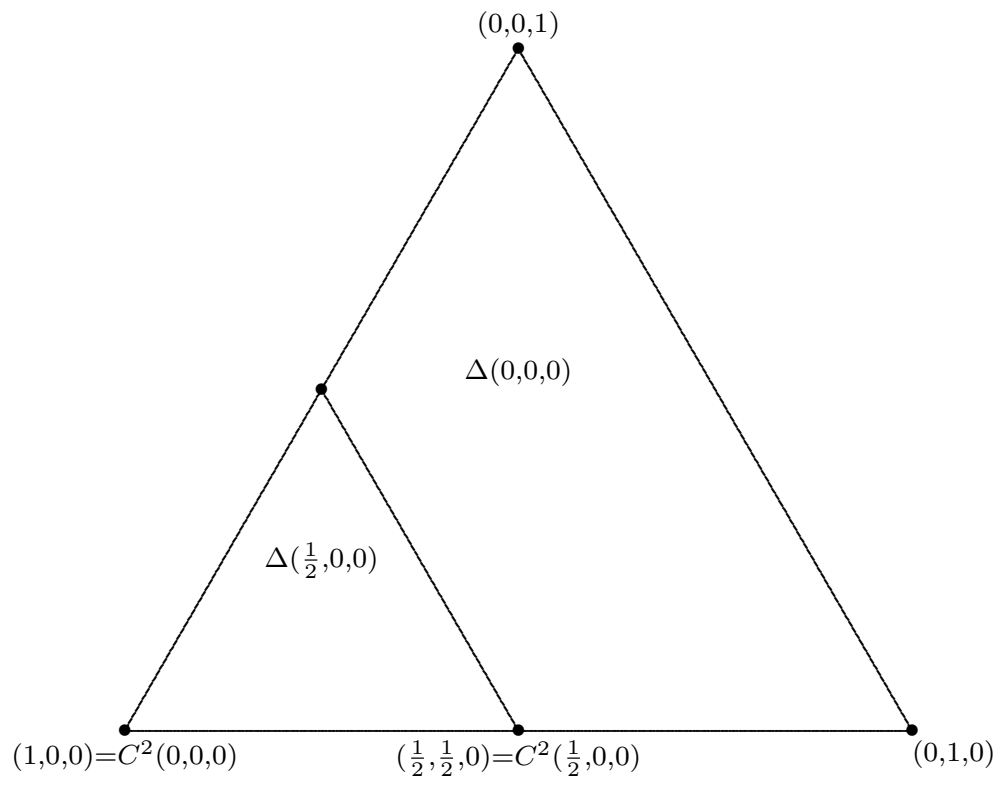

Figure 6: Theorem 4, the case $K=1$. 


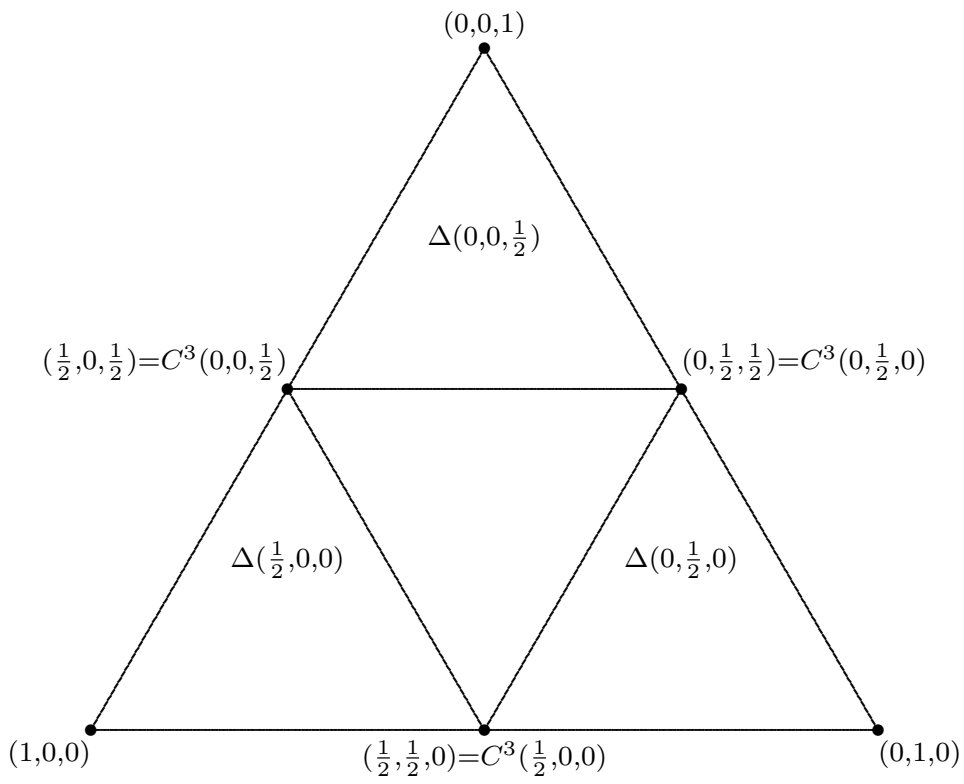

Figure 7: Theorem 4, the case $K=2$.

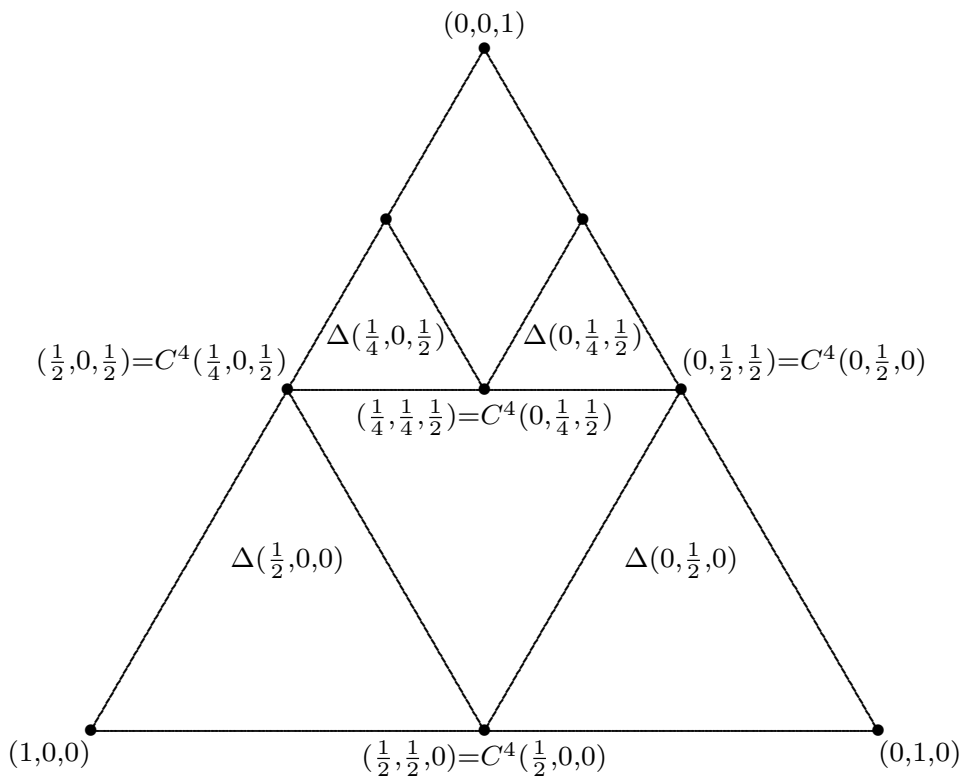

Figure 8: Theorem 4 , the case $K=3$. 


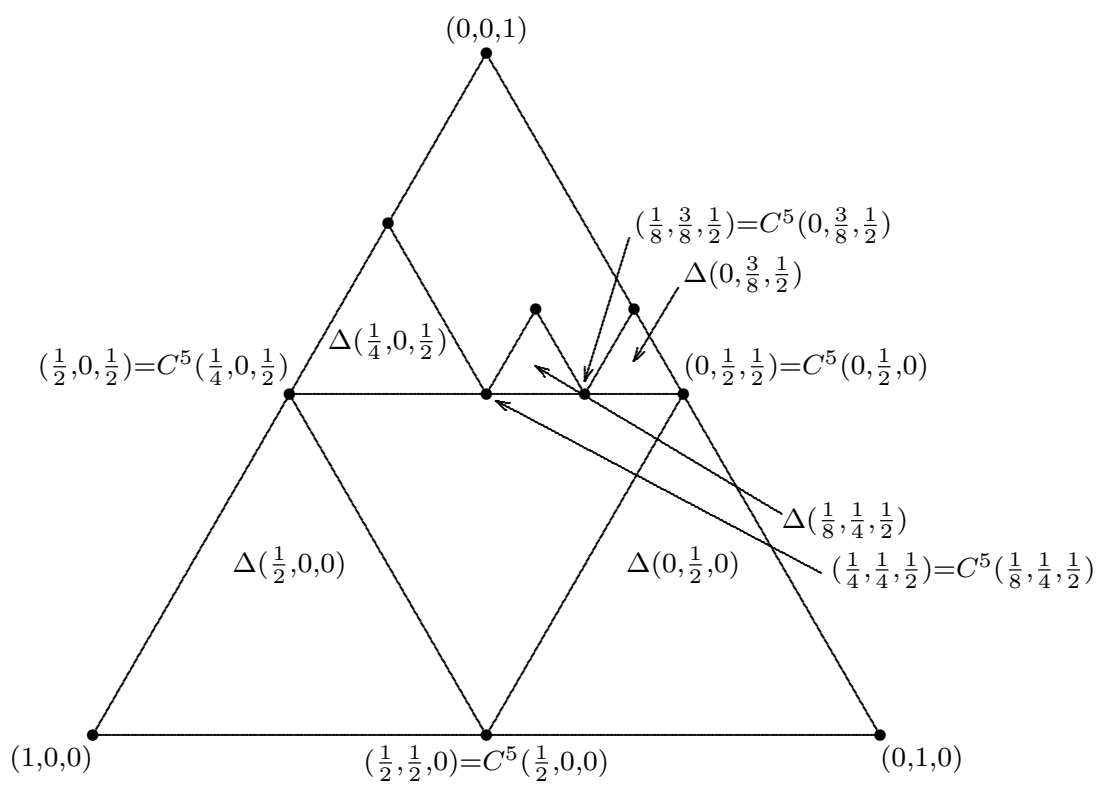

Figure 9: Theorem 4, the case $K=4$.

Even if continuity and monotonicity are added to independence of irrelevant alternatives, a choice function on the simplex domain need not be rationalizable. Let $n=3$ and consider, for example, the $N$-path choice function $C^{\Pi}$ defined by letting $p=\left(\frac{1}{3}, \frac{1}{3}, \frac{1}{3}\right)$,

$$
\begin{aligned}
& f^{1}\left(t_{1}\right)= \begin{cases}\left(t_{1}, t_{1}, 1-2 t_{1}\right) & \text { if } t_{1} \in\left[\frac{1}{3}, \frac{1}{2}\right], \\
\left(t_{1}, 1-t_{1}, 0\right) & \text { if } t_{1} \in\left(\frac{1}{2}, 1\right],\end{cases} \\
& f^{2}\left(t_{2}\right)= \begin{cases}\left(1-2 t_{2}, t_{2}, t_{2}\right) & \text { if } t_{2} \in\left[\frac{1}{3}, \frac{1}{2}\right], \\
\left(0, t_{2}, 1-t_{2}\right) & \text { if } t_{2} \in\left(\frac{1}{2}, 1\right],\end{cases} \\
& f^{3}\left(t_{3}\right)= \begin{cases}\left(t_{3}, 1-2 t_{3}, t_{3}\right) & \text { if } t_{1} \in\left[\frac{1}{3}, \frac{1}{2}\right], \\
\left(1-t_{3}, 0, t_{3}\right) & \text { if } t_{3} \in\left(\frac{1}{2}, 1\right] .\end{cases}
\end{aligned}
$$

Because $C^{\Pi}$ is an $N$-path choice function, it satisfies independence of irrelevant alternatives, continuity, and monotonicity. But this choice function is not rationalizable because there exists a cycle of length three formed by the points $\left(\frac{1}{2}, 0, \frac{1}{2}\right)=C^{\Pi}\left(0,0, \frac{1}{2}\right),\left(0, \frac{1}{2}, \frac{1}{2}\right)=$ $C^{\Pi}\left(0, \frac{1}{2}, 0\right)$ and $\left(\frac{1}{2}, \frac{1}{2}, 0\right)=C^{\Pi}\left(\frac{1}{2}, 0,0\right)$. See Figure 10 for an illustration. 


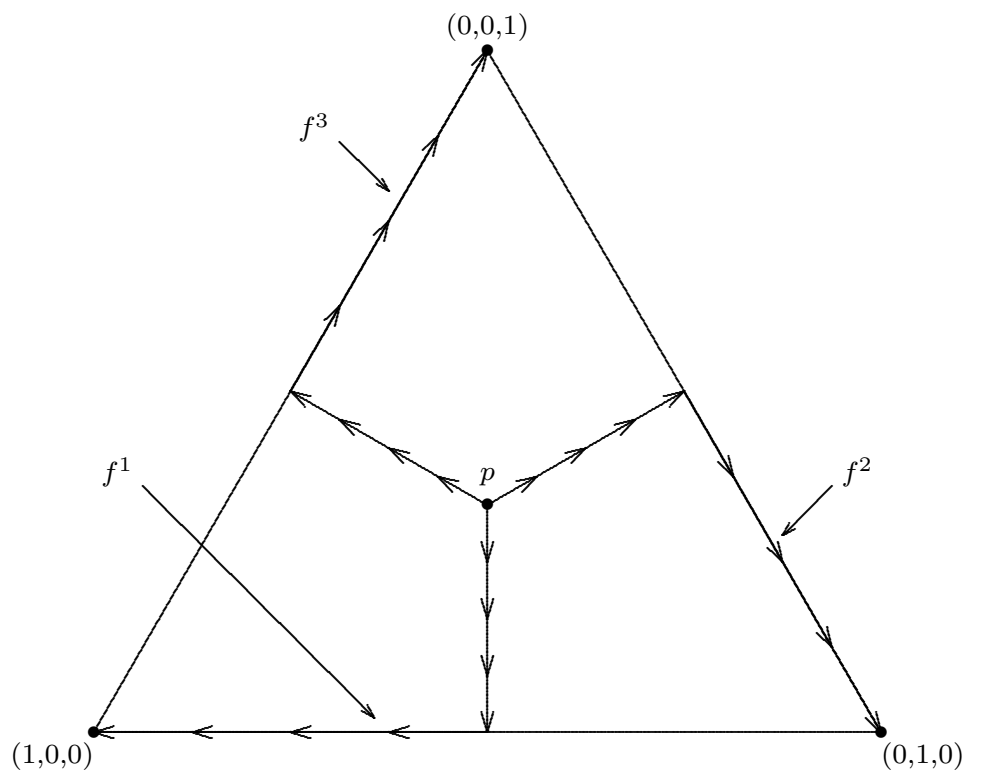

Figure 10: A non-rationalizable $n$-path choice function $C^{\Pi}$.

It is straightforward to verify that, for $n=3$, some $N$-path choice functions are rationalizable. A simple example is given by the $N$-path choice function $C^{\Pi}$ with $p=\left(\frac{1}{3}, \frac{1}{3}, \frac{1}{3}\right)$,

$$
\begin{array}{ll}
f^{1}\left(t_{1}\right)=\left(t_{1}, \frac{1-t_{1}}{2}, \frac{1-t_{1}}{2}\right) & \text { for all } t_{1} \in\left[\frac{1}{3}, 1\right], \\
f^{1}\left(t_{1}\right)=\left(t_{1}, \frac{1-t_{1}}{2}, \frac{1-t_{1}}{2}\right) & \text { for all } t_{1} \in\left[\frac{1}{3}, 1\right], \\
f^{1}\left(t_{1}\right)=\left(t_{1}, \frac{1-t_{1}}{2}, \frac{1-t_{1}}{2}\right) & \text { for all } t_{1} \in\left[\frac{1}{3}, 1\right] .
\end{array}
$$

This choice function is rationalized by the Euclidean distance from $p$ multiplied by -1 , that is, we have, for all $\alpha \in A$ and for all $x \in \Delta(\alpha)$,

$$
C^{\Pi}(\alpha)=x \Leftrightarrow\|p-x\| \leq\|p-y\| \text { for all } y \in \Delta(\alpha) .
$$

See Figure 11. 


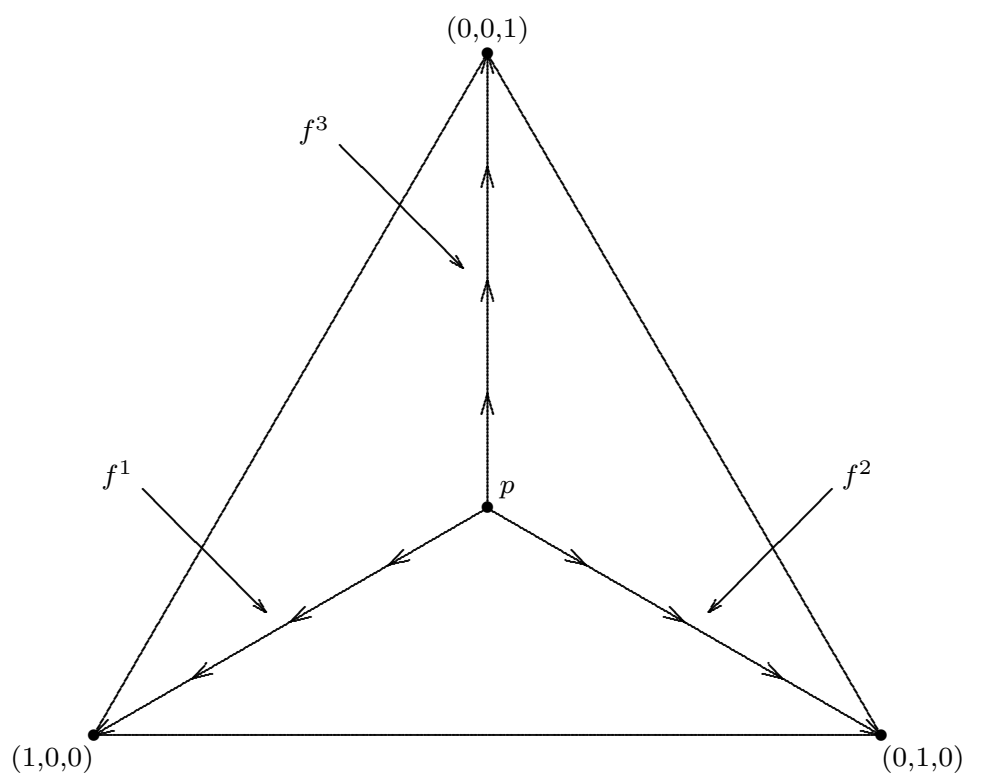

Figure 11: A rationalizable $n$-path choice function $C^{\Pi}$.

It turns out that in the presence of independence of irrelevant alternatives and continuity of the choice function, it is necessary and sufficient to exclude cycles of length three in order to obtain rationalizability. In view of Theorem 3, this statement is equivalent to the following result.

Theorem 5. Let $n=3$ and let $C=C^{\Pi}$, where $\Pi$ is a weakly monotonic $N$-path. Then $C$ satisfies the strong axiom of revealed preference if and only if there is no revealed-preference cycle of length three in $C$.

Proof. If $C$ satisfies the strong axiom of revealed preference, then clearly there is no revealed-preference cycle of length three.

Now suppose that there is no revealed-preference cycle of length three in $C$. Because the simplex domain is closed under intersection and choice functions corresponding to weakly monotonic $N$-paths satisfy independence of irrelevant alternatives, they also satisfy the weak axiom of revealed preference and, thus, there is no revealed-preference cycle of length two in $C$. Let $K>3$ and let $x^{1}, \ldots, x^{K}$ be distinct alternatives such that $x^{k} R^{C} x^{k+1}$ for all $k \in\{1, \ldots, K\}$, where $x^{K+1}=x^{1}$. The proof is complete once we establish that there is a revealed-preference cycle of length less than $K$ in $C$.

(a) Let $\alpha \in A$ such that $\Delta(\alpha)$ is the (unique) smallest choice set containing the points $x^{1}, \ldots, x^{K}$, and write $D=\Delta(\alpha)$. Then $\left\{x^{1}, \ldots, x^{K}\right\} \cap\{\ell(\alpha), r(\alpha), t(\alpha)\}=\emptyset$ since otherwise by Lemma 1 there would be a cycle of length two. Also, note that each edge of $D$ contains an element of $\left\{x^{1}, \ldots, x^{K}\right\}$ in its interior. Suppose, without loss of generality, that $x^{1}$ is in the relative interior of the left edge of $D$ and that $x_{3}^{2} \geq x_{3}^{1}$. Then by Lemma 1 we have $x^{1}=C\left(\left\{x \in D \mid x_{3} \geq x_{3}^{1}\right\}\right)$ and $x^{2} \in\left\{x \in D \mid x_{3} \geq x_{3}^{1}\right\}$. See Figure 12. If 
$\left\{x \in D \mid x_{3} \geq x_{3}^{1}\right\} \cap\left\{x^{3}, \ldots, x^{K}\right\} \neq \emptyset$ then we can construct a shorter cycle and are done. So we now assume $\left\{x \in D \mid x_{3} \geq x_{3}^{1}\right\} \cap\left\{x^{3}, \ldots, x^{K}\right\}=\emptyset$, that is, $x_{3}^{3}, \ldots, x_{3}^{K}<x_{3}^{1}$.

Next let

$$
E=\left\{x \in D \mid x_{2} \geq x_{2}^{2}\right\} .
$$

If $x^{k} \in E$ for some $k \in\{4, \ldots, K\}$, then we have $x^{2} R^{C} x^{k}$ and therefore a cycle of length less than $K$ in $C$. Now we assume that $x^{4}, \ldots, x^{K} \notin E$. Consider the parallelograms

$$
F=\left\{x \in D \mid x_{2} \leq x_{2}^{2} \text { and } x_{3} \leq x_{3}^{1}\right\}
$$

and

$$
G=\left\{x \in D \mid x_{1} \leq x_{1}^{2} \text { and } x_{3} \leq x_{3}^{1}\right\}
$$

(the shaded regions in Figure 12). Suppose $x^{2}$ is not on the right edge of $D$, that is, $G \neq \emptyset$. Since $D$ is minimal there is a $k \neq 1,2$ with $x^{k} \in G$. If $x^{k+1} \in F$, then we also have $x^{k} R^{C} x^{2}$ because $x^{k} R^{C} x^{k+1}$ and thus there is a shorter cycle. Now assume $x^{k+1} \in G$ and, by repeating this argument, $x^{K} \in G$. Since $x^{K} R^{C} x^{1}$, we obtain again $x^{K} R^{C} x^{2}$, and thus a shorter cycle.

For the remainder of the proof we assume that $x^{2}$ is on the right edge of $D$, that is, $G=\emptyset$.

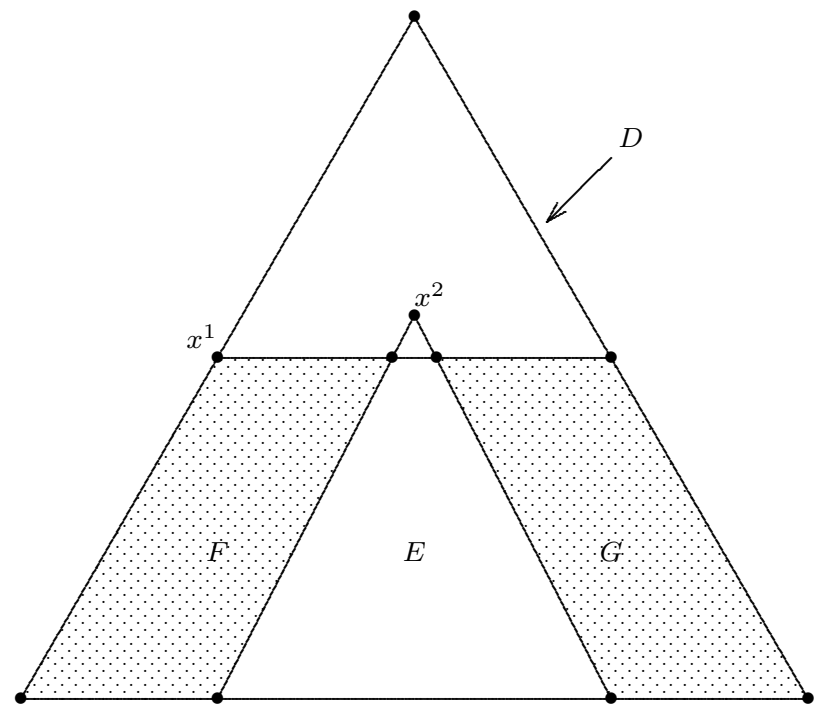

Figure 12: Theorem 5, part (a).

(b) Let

$$
H=\left\{x \in D \mid x_{1} \geq x_{1}^{1}\right\}
$$

(see Figure 13). Since $x^{2} R^{C} x^{3}$ we have $x^{3} \in E$, since otherwise by Lemma 1 we would have $x^{2} R^{C} x^{1}$, a contradiction. Consider a choice set (triangle) $D^{\prime}$ so that $x^{3} R^{C} x^{4}$. If $x^{3}$ is 
on the left edge including the left and top vertices of $D^{\prime}$, we have $x^{4} \in E$ and therefore, by Lemma $1, x^{2} R^{C} x^{4}$ and thus a shorter cycle. So we now assume that $x^{3}$ is on the right or bottom edge of $D^{\prime}$ including the right vertex. If $x^{3}$ is on the bottom edge of $D^{\prime}$ excluding the vertices, Lemma 1 implies $x^{3} R^{C} x^{1}$ and thus we have a cycle of length three, a contradiction. Hence, $x^{3}$ is on the right edge or the right vertex of $D^{\prime}$. This implies, moreover, that $x_{1}^{3}>x_{1}^{1}$; otherwise, again by Lemma 1 , we have $x^{3} R^{C} x^{1}$ and thus a cycle of length three, a contradiction. In particular, $x^{3} \in E \cap H$ and $x^{3}$ is not on the right edge or top or right vertices of $E \cap H$.

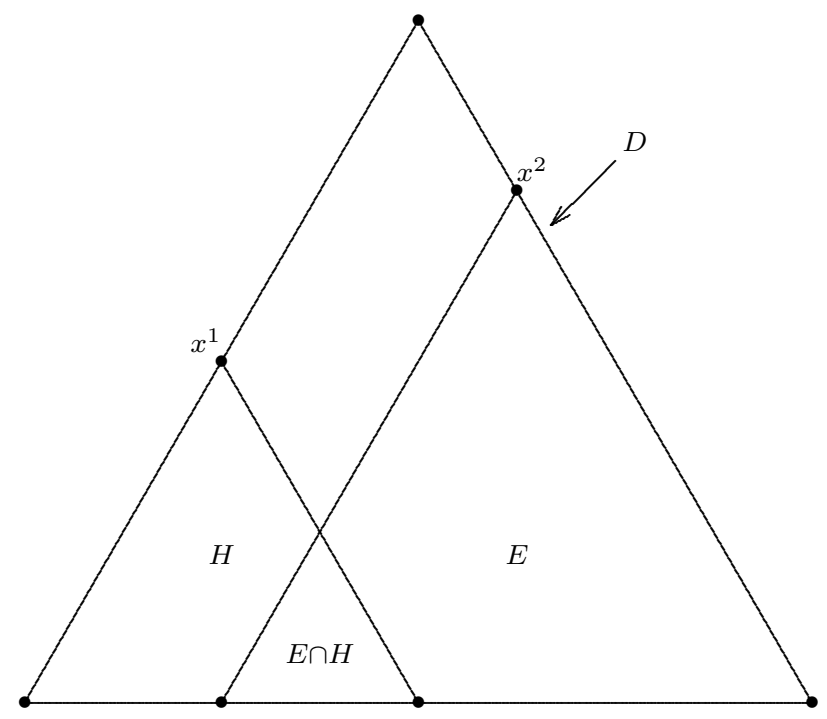

Figure 13: Theorem 5, part (b).

(c) To conclude the proof, we repeat the arguments in (a) and (b) but now for $x^{2}, \ldots, x^{5}$ instead of $x^{1}, \ldots, x^{4}$, respectively, where $x^{5}=x^{1}$ in case $K=4$. Define, analogously to $E$ and $H$, the sets $E^{\prime}$ and $H^{\prime}$ by

$$
E^{\prime}=\left\{x \in D \mid x_{1} \geq x_{1}^{3}\right\} \text { and } H^{\prime}=\left\{x \in D \mid x_{3} \geq x_{3}^{2}\right\} .
$$

Note that the arguments in (a) and (b) for $x^{1}, \ldots, x^{4}$ either resulted in shorter cycles or, in the end, in $x^{3} \in E \cap H$. Since $E^{\prime} \cap H^{\prime}=\emptyset$, analogous arguments for $x^{2}, \ldots, x^{5}$ result in shorter cycles.

\section{Concluding remarks}

The main open problem in connection to this paper is the rationalizability question if $n>3$. On the one hand, the existing literature (see Section 1) would suggest that even under additional conditions exclusion of cycles of a particular length does not imply the 
strong axiom of revealed preference. On the other hand, the domain of choice problems in the present paper is rather restricted, so that this general insight may not carry over.

Finally, we note that the axioms of independence of irrelevant alternatives and continuity are incompatible with the assumption that requires $C$ to assume all possible values in its range $\Delta(0, \ldots, 0)$. If $p_{1}=1$, the point $e^{i}$ for any $i \in\{2, \ldots, n\}$ will never be chosen, as can be seen by applying Lemma 1 ; similarly, if $p_{1} \neq 1, e^{1}$ is never chosen.

\section{References}

Arrow, K.J. (1959), Rational choice functions and orderings, Economica 26, 121-127.

Blackorby, C., W. Bossert and D. Donaldson (1995), Multi-valued demand and rational choice in the two-commodity case, Economics Letters 47, 5-10.

Bossert, W. (1994), Rational choice and two-person bargaining solutions, Journal of Mathematical Economics 23, 549-563.

Bossert, W. and H. Peters (2009), Single-peaked choice, Economic Theory 41, 213-230.

Bossert, W. and K. Suzumura (2010), Consistency, Choice, and Rationality, Cambridge, MA: Harvard University Press.

Nash, J.F. (1950), The bargaining problem, Econometrica 18, 155-162.

Peters, H. and P. Wakker (1991), Independence of irrelevant alternatives and revealed group preferences, Econometrica 59, 1787-1801.

Peters, H. and P. Wakker (1994), WARP does not imply SARP for more than two commodities, Journal of Economic Theory 62, 152-160.

Richter, M.K. (1966), Revealed preference theory, Econometrica 41, 1075-1091.

Richter, M.K. (1971), Rational choice, in: J.S. Chipman, L. Hurwicz, M.K. Richter and H.F. Sonnenschein, eds., Preferences, Utility, and Demand, New York: Harcourt Brace Jovanovich, 29-58.

Rose, H. (1958), Consistency of preference: the two-commodity case, Review of Economic Studies 25, 124-125.

Samuelson, P.A. (1938), A note on the pure theory of consumer's behaviour, Economica 5, $61-71$.

Stovall, J.E. (2014), Collective rationality and monotone path division rules, Journal of Economic Theory, 154, 1-24.

Thomson, W. (2015), Axiomatic and game-theoretic analysis of bankruptcy and taxation problems: an update, Mathematical Social Sciences, 74, 41-59.

Uzawa, H. (1960), Preference and rational choice in the theory of consumption, in: K.J. Arrow, S. Karlin and P. Suppes, eds., Mathematical Methods in the Social Sciences, 1959, Stanford: Stanford University Press, 129-148. 